non-steady layer would equal the steady layer in times given approximately by

$$
\begin{aligned}
& t \approx \frac{5 x}{U} \quad \text { for velocity, } \\
& t \approx \frac{6 x}{U} \quad \text { for temperature. }
\end{aligned}
$$

Thus to a reasonable approximation it can be said that by the time a point on a suddenly accelerated plate moves 5 times its distance from the leading edge, its boundary layers will have become steady state ones.

\title{
PLASTIC WAVE PROPAGATION IN A BAR OF MATERIAL EXHIBITING A STRAIN RATE EFFECT ${ }^{1}$
}

\author{
By L. E. MALVERN (Carnegie Institute of Technology)
}

1. Introduction. The propagation of a transient wave of plastic deformation due to longitudinal impact on a bar has been treated by Donnell, ${ }^{2}$ and White and Griffis, ${ }^{3}$ by a non-linear superposition method. The partial differential equations governing the wave propagation were derived independently by Taylor ${ }^{4}$ and von Kármán ${ }^{5}$ under the assumption of a relation between stress and strain independent of strain rate. Constant velocity tension impact tests at the California Institute of Technology ${ }^{6.7}$ gave fair agreement with the theory. Some systematic discrepancies were, however, observed. In the tension impact tests the maximum residual strain was smaller than predicted by the theory, and the observed force-time variation at the fixed end during impact showed that the stress there was greater than the theory predicted. It has been suggested $^{6}$ that these discrepancies were due to the use in the theory of an invariant relation between stress and strain independent of strain rate. At the high strain rates involved in deformation under impact a considerable deviation from the static stress-strain relation may be expected. The present work extends the theory to apply to materials in which the stress is a function of the instantaneous plastic strain and strain rate.

\footnotetext{
${ }^{1}$ Received June 5, 1950. The results presented here were obtained in the course of research conducted at Brown University under Contract N7onr-358 sponsored jointly by the Office of Naval Research and the Bureau of Ships. This paper is part of a thesis submitted in partial fulfillment of the requirements for the degree of Doctor of Philosophy at Brown University, October, 1949.

${ }^{2}$ L. H. Donnell, Longitudinal wave transmission and impact, A.S.M.E., Trans. 52 (1), APM 153-167 (1930).

${ }^{3}$ M. P. White and L. Griffis, The permanent strain in a uniform bar due to longitudinal impact, J. Appl. Mech., A.S.M.E., Trans. 69, A-337-A-343 (1947).

${ }^{4}$ G. I. Taylor, Propagation of earth waves from an explosion, British Official Report R.C. 70 (1940).

${ }^{5}$ Th. v. Kármán, On the propagation of plastic deformation in solids, N.D.R.C. Report No. A-29 (O.S.R.D. No. 365) (1942).

${ }^{6}$ P. E. Duwez, D. S. Wood, D. S. Clark, and J. V. Charyk, The effect of stopped impact and reflection on the propagation of plastic strain in tension, N.D.R.C. Report No. A-108, (O.S.R.D. No. 988) (1942).

'P. E. Duwez and D. S. Clark, An experimental study of the propagation of plastic deformation under conditions of longitudinal impact, A.S.T.M., Proc. 47, 502-532 (1947).
} 
2. Proposed flow law for tension and compression. It is assumed that in longitudinal impact on a cylindrical.or prismatic bar a relation of the form

$$
\sigma=\phi\left(\epsilon^{\prime \prime}, \epsilon^{\cdot \prime \prime}\right)
$$

exists between the values of the nominal tensile stress $\sigma$ (longitudinal force per unit of initial cross-sectional area), plastic strain $\epsilon^{\prime \prime}$ (permanent change in length per unit initial length), and the plastic strain rate $\epsilon^{\prime \prime \prime}$. Since $\phi$ is in general an increasing function of $\epsilon^{\prime \prime}$ this determines $\epsilon^{\prime \prime}$ as a function of $\sigma$ and $\epsilon^{\prime \prime}$. This relation may be expressed as

$$
E_{0} \epsilon^{\prime \prime}=g(\sigma, \epsilon),
$$

where the factor $E_{0}$ is Youngs modulus and $\epsilon$ is the total strain. Elastic deformation is assumed to be independent of strain rate. Thus, if $\epsilon^{\prime}$ denotes the elastic strain,

$$
E_{0} \epsilon^{\prime \prime}=\sigma^{\circ} .
$$

The relation between total strain, strain rate, and stress is then

$$
E_{0} \epsilon^{\cdot}=\sigma^{\cdot}+g(\sigma, \epsilon) .
$$

The static stress-strain relation $\sigma=f(\epsilon)$ is interpreted as a succession of equilibrium states so that plastic flow occurs only when the plasticity condition

$$
\sigma>f(\epsilon)
$$

is satisfied. Otherwise the elastic law (2) applies instead of the plastic flow law (3). The elastic law also applies until the initial yield strain $\epsilon_{y}$ is reached on the first loading. The plasticity condition (4) as stated applies to tensile impact ( $\sigma$ and $\epsilon$ positive) but the same form of law may be used in compressive impact if compressive stress and strain are reckoned positive.

There is some evidence that the right-hand member of (1) should have the form of $f(\epsilon)$ plus a term depending logarithmically on the plastic strain rate. ${ }^{8}$ If this is the case $g(\sigma, \epsilon)$ will depend exponentially on $\sigma-f(\epsilon)$, the excess of the instantaneous stress over the static stress at the same strain. Sokolovsky ${ }^{9}$ has treated wave propagation in a material without work-hardening using a law of the form (3) in which $g\left(\sigma-\sigma_{\nu}\right)$ was a function only of the excess of the stress over the initial yield stress $\sigma_{\nu}$.

3. Equations governing the wave propagation. The propagation of the wave of plastic deformation is governed by the following system of three partial differential equations, in which $x$ denotes the initial distance of a cross section from the impact end, $v$ the particle velocity (assumed constant over each cross section, and $\rho$ the initial density.

$$
\begin{gathered}
\frac{\partial \sigma}{\partial x}-\rho \frac{\partial v}{\partial t}=0, \\
\frac{\partial \epsilon}{\partial t}-\frac{\partial v}{\partial x}=0, \\
E_{0} \frac{\partial \epsilon}{\partial t}-\frac{\partial \sigma}{\partial t}=g(\sigma, \epsilon) .
\end{gathered}
$$

${ }^{8}$ See, for example, H. Deutler, Experimentelle Untersuchung ueber die Abhaengigkeit der Zugspannungen von der Verformungsgeschwindigkeit, Phys. Z. 33, 247-259 (1932).

${ }^{9} \mathrm{~V}$. V. Sokolovsky, The propagation of elastic-viscous-plastic waves in bars, Prikl. Mat. i Mek. 12, 261-280 (1948). 
The first equation is the longitudinal equation of motion. The second equation is a consequence of the fact that $v=\partial u / \partial t$ and $\epsilon=\partial u / \partial x$, where $u(x, t)$ is the displacement at time $t$ of the cross section which was initially at distance $x$ from the impact end of the bar, and the third equation is the law (3).

The system (5) is a hyperbolic system of quasi-linear partial differential equations which may be integrated numerically by the method of characteristics under appropriate boundary conditions. The characteristics in the $x, t$-plane are the three families of straight lines defined by the characteristic differential equations

$$
d x=0, \quad d x-c_{0} d t=0, \quad d x+c_{0} d t=0,
$$

where

$$
c_{0}=\left(E_{0} / \rho\right)^{1 / 2}
$$

is the constant speed of propagation of longitudinal elastic waves in the bar.

The following three equations hold respectively along the three characteristics defined by (6).

$$
\begin{aligned}
E_{0} d \epsilon-d \sigma & =g(\sigma, \epsilon) d t \\
d \sigma-\rho c_{0} d v & =-g(\sigma, \epsilon) d t \\
d \sigma+\rho c_{0} d v & =-g(\sigma, \epsilon) d t
\end{aligned}
$$

The equations (6) may be integrated immediately to give the fixed straight characteristics of the plastic region of the $x, t$-plane, but the equations (7) will in general require

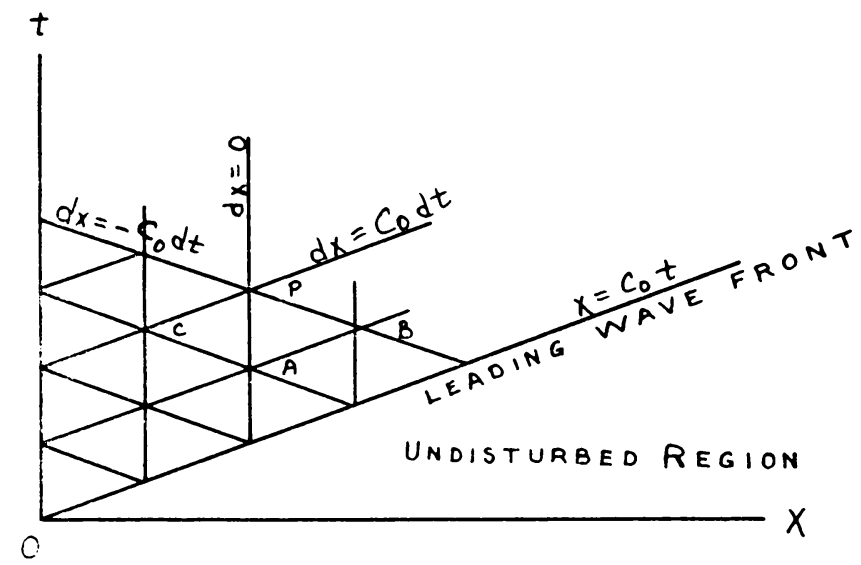

FIG. 1 Characteristics in the $x, t$-plane.

step-by-step numerical integration. For this purpose the differentials of (7) are replaced by finite differences, and the value of $g(\sigma, \epsilon)$ in each equation is taken as an average of the values along the appropriate segment of the characteristic.

4. Boundary conditions for continued impact on a semi-infinite bar. Consider a continued tensile impact on a semi-infinite bar which is initially at rest. The extension of the theory to finite bars and finite durations of impact is not difficult, although the 
numerical integration becomes more involved. The $x$-axis is chosen so that at the instant of impact, the impact end is at the origin and the bar lies along the positive $x$ ax ${ }_{1}$ s. Suppose that at time $t=0$, the end of the rod is instantaneously set in motion with velocity $v_{0}$ such that $\left|v_{0}\right|>c_{0} \epsilon_{y}$ after which the end velocity is maintained constant at the value $v_{0}$. For a tensile impact the velocity is negative.

Immediately upon impact a shock wave of elastic deformation begins to travel along the bar at the speed $c_{0}$. This leading wave front is represented in Fig. 1 by the line $x=c_{0} t$. The shock wave conditions which hold across an elastic shock wave traveling in the positive direction are

$$
\begin{aligned}
& \Delta \sigma=-\rho c_{0} \Delta v, \\
& \Delta v=-c_{0} \Delta \epsilon, \\
& \Delta \sigma=\rho c_{0}^{2} \Delta \epsilon=E_{0} \Delta \epsilon,
\end{aligned}
$$

where $\Delta \sigma, \Delta \epsilon$, and $\Delta v$ are the jumps in stress, strain, and velocity, respectively, as the shock wave passes. The first condition results from equating impulse to change of momentum for the traversing of an element of the bar by the shock wave. The second condition is a consequence of the continuity of displacement across the shock and the third condition follows from the first two.

Since $\sigma=\epsilon=v=0$ in the undisturbed region ahead of the shock wave, the jump conditions (8) yield

$$
\sigma=\rho c_{0}^{2} \epsilon=-\rho c_{0} v \quad \text { on } \quad x=c_{0} t
$$

just after the shock wave passes. The line $x=c_{0} t$ is also a characteristic of (5) along which the second equation of (7) holds. This equation may be integrated after eliminating $\epsilon$ and $v$ by use of (9) to yield

$$
\int_{\sigma_{0}}^{\sigma} \frac{d \tau}{g\left(\tau, \tau / \rho c_{0}^{2}\right)}=-\frac{1}{2} t
$$

where $\sigma_{0}=-\rho c_{0} v_{0}$ is the stress at $x=0, t=0$. Equations (8) and (10) thus determine $\sigma, \epsilon$, and $v$ along $x=c_{0} t$. With this information and the boundary condition $v=v_{0}$ on $x=0$, the numerical integration of the equations (7) may be performed to determine $\sigma, \epsilon$ and $v$ throughout the plastic region. The values so obtained should be checked with the plasticity condition (4) at each point to make sure that the point is in the plastic region.

5. Impact of finite duration: unloading. If the impact is of duration $t_{0}$, after which the boundary condition is $\sigma=0$ on $x=0$, the solution may be constructed as in the preceding section up to the characteristic of the family $d x-c_{0} d t=0$ passing through the point $\left(0, t_{0}\right)$, i.e. up to the line $M N$ of Fig. 2. In the unloading region Eq. (2) replaces Eq. (3) so that $g(\sigma, \epsilon) \equiv 0$ for unloading. Equations $(6,7)$ then yield

$$
\begin{aligned}
& \sigma-E_{0} \epsilon=\text { const. } \quad \text { on } \quad x=\text { const., } \\
& \sigma-\rho c_{0} v=\text { const. } \quad \text { on } \quad x-c_{0} t=\text { const., } \\
& \sigma+\rho c_{0} v=\text { const. } \quad \text { on } \quad x+c_{0} t=\text { const. }
\end{aligned}
$$


The various constants are determined by matching solutions at the elastic-plastic unloading boundary in the $x, t$-plane.

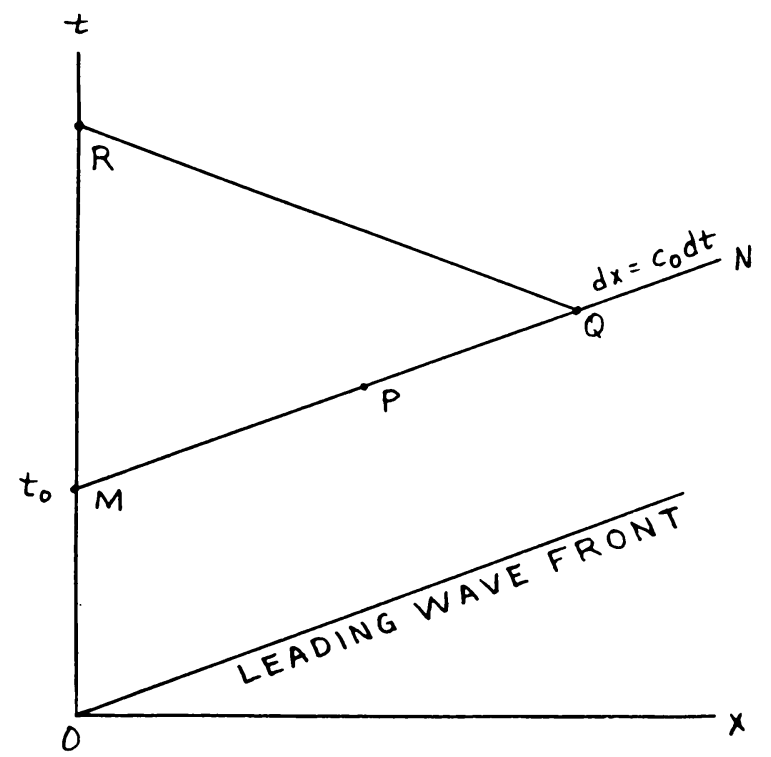

FIg. 2 Unloading shock wave $M N$ after impact of duration $t_{0}$ on a semi-infinite bar.

A sudden reduction of the impact end stress to zero causes the initial unloading wave to be a shock wave. Since the shock wave is elastic the jump conditions (8) apply and the wave travels at the speed $c_{0}$, i.e. along $M N$, Fig. 2 . The shock intensity decreases as the wave progresses and may decrease to zero. If the shock wave travels as far as $Q$, Fig. 2, the solution is determined in the triangle $M Q R$ by Eqs. (11), the boundary condition $\sigma=0$ on $x=0$, and the jump conditions (8) together with the known values of $\sigma, \epsilon$, and $v$ along $M N$ before the shock wave passes.

If the shock wave is absorbed, say at $Q$, the elastic-plastic boundary becomes a boundary of continuous transition from the plastic to the elastic state, and does not in general continue along the line $M N$. Along a continuous unloading boundary the static relation $\sigma=f(\epsilon)$ holds. This condition and the characteristic conditions in the elastic and plastic regions suffice to determine the position of the unloading boundary, although a trial and error procedure is usually needed to find the boundary points. A continuous transition from plastic to elastic behavior may begin even before the impact ends. The unloading shock wave then travels through an elastic region until it overtakes the continuous unloading boundary.

6. Solutions of the equations. Numerical integration of the system (5) has been carried out for an idealized form of the law (3) in which $g(\sigma, \epsilon) \equiv k[\sigma-f(\epsilon)]$ where $k$ is a multiplicative constant, with the function $f(\epsilon)$ chosen in a simple form which approximates the static stress-strain curve of a hardened aluminum. Even with the idealized law used the solutions indicate that a law of the type (3) can account for the discrepancies observed in the stress-time variation at the fixed end of impact specimens. This type of law does not, however, account for the discrepancy observed in the maximum residual strain. 
It was possible to obtain an explicit solution for the equations in the case of a non work-hardening material of a type previously treated numerically by Sokolovsky. ${ }^{9}$ With $g(\sigma, \epsilon) \equiv k\left(\sigma-\sigma_{\nu}\right)$, where $\sigma_{\nu}$ is the yield stress, and with the introduction of the dimensionless variables

$$
\begin{array}{ll}
T=k t & X=x k / c_{0} \\
S=\sigma / \sigma_{\nu} & E=\epsilon / \epsilon_{\nu} \quad V=v / c_{0} \epsilon_{\nu},
\end{array}
$$

the system (5) becomes

$$
\begin{aligned}
& \frac{\partial S}{\partial X}=\frac{\partial V}{\partial T}, \\
& \frac{\partial E}{\partial T}=\frac{\partial V}{\partial X} \\
& \frac{\partial E}{\partial T}=\frac{\partial S}{\partial T}+S-1 .
\end{aligned}
$$

Elimination of $E$ and $V$ yields

$$
\frac{\partial^{2} S}{\partial X^{2}}-\frac{\partial^{2} S}{\partial T^{2}}-\frac{\partial S}{\partial T}=0 .
$$

The boundary conditions for $S$ in the plastic region of the $X, T$-plane (above $X=T$ ) are

$$
\begin{gathered}
\frac{\partial S}{\partial X}=0 \quad \text { on } \quad X=0 \\
S=1+C \exp (-T / 2) \quad \text { on } \quad X=T,
\end{gathered}
$$

where

$$
C=\left|V_{0}\right|-1=\left|v_{0} / c_{0} \epsilon_{y}\right|-1 .
$$

Introduction of the canonical variables, $\alpha=\frac{1}{2}(T+X)$ and $\beta=\frac{1}{2}(T-X)$ and a new function $P$ defined by

$$
S=1+C P \exp \left[-\frac{1}{2}(\alpha+\beta)\right]
$$

yields the telegraph equation for $P$,

with boundary conditions

$$
\frac{\partial^{2} P}{\partial \alpha \partial \beta}-\frac{1}{4} P=0
$$

$$
\begin{array}{rlrlrl}
\frac{\partial P}{\partial n}=0 & \text { on } & \alpha & =\beta \\
P & =1 & \text { on } & & \beta=0
\end{array}
$$

By a Laplace transform technique, or by the Riemann method of integration it may be determined that

$$
P(\alpha, \beta)=I_{0}\left[(\alpha \beta)^{1 / 2}\right]
$$


is the solution required, where $I_{0}$ is the Bessel function of order zero with pure imaginary argument. Thus, returning to the variables, $X$ and $T$, the dimensionless stress solution is

$$
S(X, T)=1+C I_{0}\left[\frac{1}{2}\left(T^{2}-X^{2}\right)^{1 / 2}\right] \exp (-T / 2) .
$$

The strain and velocity solutions are then obtained from the characteristic conditions by numerical quadratures.

\section{BOOK REVIEWS}

\section{Nonlinear vibrations in mechanical and electrical systems. By J. J. Stoker, Interscience Publishers, Inc., New York, 1950. xv + 273. \$5.00.}

Chapter I contains a concise review of linear vibrations in one degree of freedom. This is followed, in Chapter II, by a discussion of free vibrations of undamped systems with nonlinear restoring forces. One finds here, in addition to the material given in the books by Timoshenko (Vibrations Problems in Engineering. D. Van Nostrand Co., N. Y., 1937) and Den Hartog (Mechanical Vibrations. McGraw-Hill Book Co., N.Y., 1940) the notion of the energy curves in the phase plane. The theory of this chapter is illustrated by interesting examples.

Chapter III is divided into three sections; the first explains Liénhard's graphical construction of trajectories in the phase plane, the second gives the theory of singular points of first order differential equations, and the third shows applications of that theory to nonlinear mechanical and electromechanical systems. A good deal of attention is devoted to a novel treatment of the elastic stability of columns from a dynamical viewpoint, making use of the notion of singular points.

Chapter IV deals essentially with Duffing's equation and its integration by both an iteration and a perturbation method. In this chapter reliance is placed on physical intuition, the more delicate questions of the existence of the perturbation series, and the stability of solutions being left for later treatment. A special case of subharmonic resonance in Duffing's equation with and without damping is discussed, and Rauscher's iteration method is briefly described. Following Rauscher's method, combination tones are introduced, i.e., response frequencies which are linear combinations of the frequencies $\omega_{1}$ and $\omega_{2}$ of a forcing function $H_{1} \cos \omega_{1} t+H_{2} \cos \omega_{2} t$. The chapter ends with some introductory remarks regarding the stability of harmonic solutions and with a helpful table summarizing the essential differences in the response of Duffing's equation and of the related linear equation.

Chapter V treats, in two sections, Van der Pol's equation without and with forcing function. In the first part the autonomous equation is discussed and a very appealing treatment of relaxation oscillations, and also a higher approximation are included. The second part deals with Van der Pol's method applied to the non-autonomous system and with the stability investigations by Andronow and Witt. It gives altogether a very complete treatment for the two cases of a forcing frequency near the natural frequency of the system, and not near that frequency.

Chapter VI, the last chapter of the book, treats linear equations with periodic coefficients because of their applicability to investigations of the stability of solutions to nonlinear problems. The Floquet theory is well reproduced as is an interesting and simple discussion of the stability of the Mathieu equation $w^{\prime \prime}+(\delta+\epsilon \cos z) w=0$ in the neighborhood of $\epsilon=0$. Finally, the stability of harmonic solutions of Duffing's equation without damping is investigated by the theory of this chapter.

This final chapter is followed by six appendices giving, in that order, (1) the mathematical justification of the perturbation method, (2) the existence of combination oscillations, (3) the existence of limit cycles in self-excited systems, (4) a proof of a previously introduced and attractive argument regarding the form of the limit cycle of $d v / d \xi=\epsilon^{2}[F(v)-\xi] / v$ for a special form of $F(v)$, and as $\epsilon \rightarrow \infty$ (called the limit of limit cycles), (5) Poincaré's criterion for orbital stability, and (6) a very palatable proof of uniqueness of a limit cycle in the free oscillations of a self-sustained system. The last chapter and the appendices are distinctly not elementary in character. 